\title{
The First Run Preceded by a Quota*
}

\section{A. J. Goldman and B. K. Bender}

(March 20, 1962)

\begin{abstract}
Let $a, b, c$ be positive integers with $b \leq \min (a, c-1)$. In an infinite sequence of independent trials with different success probabilities, define a "run" to consist of a consecutive successes, and a "quota" to consist of $c$ or fewer consecutive trials containing $b$ or more successes. Recursion formulas are derived for the probabilities governing the first appearance of a run immediately preceded by a quota.
\end{abstract}

\section{Introduction}

This paper deals with the following problem, which arises in the analysis of certain targetdetection systems. Consider an infinite sequence $\left\{T_{n}: n=1,2, \ldots\right\}$ of independent probabilistic "trials." $T_{n}$ yields a "success" with probability $p_{n}$, and a "failure" with probability $q_{n}=1-p_{n}$. A run is defined to be a set of $a$ consecutive trials all yielding successes, where $a$ is a positive integer specified in advance. A quota is defined to be a set of $c$ consecutive trials, of which at least $b$ yield successes; ${ }^{1}$ here $b$ and $c$ are positive integers specified in advance, with $b<c$. When does the first run which immediately follows a quota occur?

Of course the question can only be answered on a probabilistic basis, i.e., one seeks the probability, as a function of $n$, that $T_{n}$ is the last trial of the first such run. The analysis given below leads to a recursion equation for these probabilities (more precisely, for certain other closely related ones) under the additional hypothesis that $a \geq b$. The equation is somewhat unpleasant looking, but offers no particular difficulty as regards implementation on a digital computer. ${ }^{2}$ Both the analysis and its result appear likely to be more complicated if $a<b$, so that it is fortunate that the hypothesis $a \geq b$ covers the applications we have in mind.

The special case $a=2, b=1, c=2$ has been solved by E. Brookner as part of a larger study ; ${ }^{3}$ the first author appreciates access to an advance copy of Brookner's paper. Since the methods used below are rather ad hoc, it should be noted that other work by Brookner ${ }^{4}$ provides, in principle, a systematic approach to such problems; in particular, his methods can apparently be used to derive a solution in the case $a<b$.

The next section contains a precise statement of the problem, and a guide to the main results of the investigation.

\section{Notation and Summary of Results}

The probabilistic events of interest will be regarded as subsets of a sample space, ${ }^{5}$ and so the notation of Boolean set-algebra will be employed. Thus the symbols $U$ and $\cap$ stand for set-theoretic union and intersection, respectively, while $A^{\prime}$ will generically denote the complement of the event $A$. We depart from standard notation by using the symbols $\Sigma$ and " + " for the set-theoretic union of disjoint events (i.e., "events whose probabilities can simply be summed"). We set $p_{n}=0$ for $n<1$.

\footnotetext{
*Sponsored in part by the North American Air Defense Command (Directorate of Operations Analysis) and U.S. Army Air Defense Command.

1 This definition must be slightly modified near the beginning of the sequence; see the definition of $Q_{n}$ in section 2.

2 This assumes that a routine for computing the sequence $\left\{p_{n}\right\}$ is available.

${ }^{3}$ Eli Brookner, Cumulative probability of target detection for pulsed surveillance radars, Columbia University Electronics Research Laboratories Tech. Rept. T-10/122.

${ }^{4}$ Eli Brookner, Determination of the statisties of a recurrent event in a Markov chain, Columbia University Electronies Research Laboratory unpublished manuseript.

${ }^{5}$ For this approach to probabilistic events, see e.g., the standard reference by W. Feller, An introduction to probability theory and its applications (John Wiley \& Sons, Inc., New York, N.Y.).
} 
A set-theoretic union over an empty set of indices will be taken, by convention, to be the null event $\phi$; similarly a numerical sum over an empty set of indices will be taken as zero. A set-theoretic intersection over an empty set of indices will be taken as the "sure event" (i.e., the entire sample space), while a product over an empty set of indices will be assigned the value unity.

The following events will be involved in the analysis:

$$
\begin{aligned}
& S_{n}: T_{n} \text { yielded a success }\left(S_{n}=\phi \text { for } n<1\right), \\
& S_{n}^{\prime \prime}: T_{n} \text { yielded a failure, } \\
& R_{n}=\bigcap_{0}^{a-1} S_{n-j}: T_{n} \text { is the last trial of a } \operatorname{run}\left(\text { of } a \text { consecutive successes }{ }^{6}\right. \text { ) }
\end{aligned}
$$

For the exact definition of "quota," it is convenient to define

$$
m(n)=\max (n-c+1,1) .
$$

Then we have the definitions

$Q_{n}$ : at least $b$ trials among $T_{n}, T_{n-1}, \ldots, T_{m(n)}$ yield successes,

$E_{n}=R_{n} \cap Q_{n-a}$ : the type of event whose first occurrence interests us.

Verbally, $E_{n}$ occurs if the $a$ trials numbered $n, n-1, \ldots, n-a+1$ all yield successes, and in addition $b$ or more of the $\min (c, n-a)$ trials numbered $n-a, n-a-1, \ldots, m(n-a)$ yield successes. Still other events will be required and introduced later.

The generic notation $P(A)$ will be used for the probability of an event $A$. The probabilities of interest, those referring to the first trial which ends a run immediately preceded by a quota, are therefore designated

$$
X_{n}=P\left(E_{n} \cap \bigcap_{i=1}^{n-1} E_{i}^{\prime}\right)
$$

It will be simpler to deal, instead, with the probability

$$
Y_{n}=P\left(\bigcap_{i=1}^{n} E_{i}^{\prime}\right)
$$

that no run preceded by a quota occurs up to and including the trial $T_{n}$. The sequences $\left\{X_{n}\right\}$ and $\left\{Y_{n}\right\}$ are related by

$$
Y_{n}=1-\sum_{i=1}^{n} X_{i}, \quad X_{n}=Y_{n-1}-Y_{n}
$$

and the sequence $\left\{Y_{n}\right\}$ clearly obeys the "initial conditions"

$$
\begin{gathered}
Y_{n}=1 \quad \text { if } n<a+b, \\
Y_{a+b}=1-\prod_{i=1}^{a+b} p_{i} .
\end{gathered}
$$

We therefore assume $n>a+b$ in all that follows.

The reader is exhorted to aid himself, in following the derivations, with the diagram of an axis on which points corresponding to $T_{n}, T_{n-a+1}, T_{n-a}, T_{n-a-c+b+1}, T_{n-a-c+b}$ and $T_{n-a-c+1}$ are marked off.

The ultimate goal is a recursion equation for the sequence $\left\{Y_{n}\right\}$, while the method is the decomposition of $\bigcap_{i=1}^{n} E_{i}^{\prime}$ into a union of disjoint events, each the intersection of certain independent events. ${ }^{7}$ This analysis is carried out in section 3. The desired recursion equation,

\footnotetext{
6 This run may be imbedded in a still longer sequence of consecutive successes.

7 If the set of trials whose outcomes determine whether or not an event $A$ occurs is disjoint from the corresponding set of trials for event $B$, then $A$ and $B$ are clearly independent. This sufficient condition for independence is the one implicitly appealed to throughout the paper.
} 
for $a+b<n \leq 2 a+c$, is derived without the assumption $a \geq b$ and is given in eq (24). For $n>2 a+c$, under the assumption $a \geq b$, it is given in eq (27). Both of these equations involve as coefficients certain auxiliary probabilities whose calculation is the subject of section 6 . As for the case $n>2 a+c$ and $a<b$, which is not solved in this paper, our best progress to date is reported in eq (31).

The assumption $a \geq b$ is made throughout sections 4,5 , and 7 . In section 4 it is shown that the original recursion equation can be replaced by one which has higher order but fewer nonzero terms, and so may be more suitable for computation. The new equation is given by eq (34), which is valid for $n>2 a+c$ and $b>1$, by eq (34a), which is valid for $a+b<n<2 a+c$ and $b>1$, by eq $(34 \mathrm{~b})$, which is valid for $n>2 a+c$ and $b=0$, and by eq (34c), which is valid for $n>2 a+c$ and $b=1$. The remaining cases $(n=2 a+c$, or $n<2 a+c$ and $b \leq 1)$ are left to the reader.

Section 5 contains an examination of the special case in which $p_{n}$ is independent of $n$. The general recursion eq (24) becomes eqs (37) and (38), while the general recursion eq (27) becomes eq (39); for $b>1$, the general eq (34) yields eq (40). For this special case (i.e., $p_{n}$ $=p$ and $b>1$ ) a method is given for calculating the mean occurrence time for the first run preceded by a quota; this is given compactly as eq (47). The extension to $b \leq 1$ offers no difficulty.

In section 7 we study the probability $Z_{n}$ that the first run preceded by a quota, if it ends at $T_{n}$, occurred as a sequence of $a+b$ consecutive successes. (Note that this is not the same as the probability that the first sequence of $a+b$ consecutive successes ends at $T_{n}$.) Equation (57), which is valid for $n>a+c+1$ and $b>0$, shows how the sequence $\left\{Z_{n}\right\}$ is determined by the sequences $\left\{X_{n}\right\}$ and $\left\{Y_{n}\right\}$. The illustrative case $b=1, c=2$ is investigated in some detail, and bounds on $Z_{n}$ are found in terms of $p_{n}$; see e.g., eq (61), valid for $n>3 a+3$ and $a>1$, and eqs (62) and (63), valid for $n>6$ and $a=1$.

\section{Analysis}

In this section the hypothesis $a \geq b$ is not at first assumed. From $E_{n}=R_{n} \cap Q_{n-a}$, one has

$$
E_{n}^{\prime}=R_{n}^{\prime}+\left(R_{n} \cap Q_{n-a}^{\prime}\right),
$$

where the convention for "disjoint union" has been used. Thus

$$
\bigcap_{i=1}^{n} E_{i}^{\prime}=E_{n}^{\prime} \cap \bigcap_{i=1}^{n-1} E_{i}^{\prime}=\left(R_{n}^{\prime} \cap \bigcap_{i=1}^{n-1} E_{i}^{\prime}\right)+\left(R_{n} \cap Q_{n-a}^{\prime} \cap \bigcap_{i=1}^{n-1} E_{i}^{\prime}\right) .
$$

The first disjunct on the right in eq (7) will now be decomposed further. Let $R_{n k}^{\prime}$ be the event that the first failure among trials $T_{n}, T_{n-1}, \ldots$. (in that order) occurs at $T_{n-k}$. Then $R_{n}^{\prime}=\sum_{k=0}^{a-1} R_{n k}^{\prime}$, which implies that ${ }^{8}$

$$
R_{n}^{\prime} \cap \bigcap_{i=1}^{n-1} E_{i}^{\prime}=\sum_{k=0}^{a-1}\left(R_{n k}^{\prime} \cap \bigcap_{i=1}^{n-1} E_{i}^{\prime}\right)
$$

However, it is easy to show the inclusion

$$
R_{n k}^{\prime} \subset E_{i}^{\prime} \quad \text { for } n-k \leq i \leq n-1,1 \leq k \leq a-1 \text {; }
$$

for if $R_{n k}^{\prime}$ occurs then so does $S_{n-k}^{\prime}$, and obviously this precludes the occurrence of $R_{i}$ (and thus of $E_{i}$ ) for $n-k \leq i$ and $k \leq a-1$. By (8) and (9), we have the desired decomposition ${ }^{8 a}$

\footnotetext{
8 The distributive law of Boolean set-algebra is to be used. It will be employed at other points without explicit mention.

8a Note the use, for $k=0$, of our convention about intersections over an empty set of indices.
} 


$$
\begin{aligned}
R_{n}^{\prime} \cap \bigcap_{i=1}^{n-1} E_{i}^{\prime} & =\sum_{k=0}^{a-1}\left(R_{n k}^{\prime} \cap \bigcap_{i=n-k}^{n-1} E_{i}^{\prime} \cap \bigcap_{i=1}^{n-k-1} E_{i}^{\prime}\right) \\
& =\sum_{k=0}^{a-}\left(R_{n k}^{\prime} \cap \bigcap_{i=1}^{n-k-1} E_{i}^{\prime}\right) .
\end{aligned}
$$

Now $R_{n k}^{\prime}$ and $\bigcap_{i=1}^{n-k-1} E_{?}^{\prime}$ are independent, and furthermore

$$
P\left(R_{n k}^{\prime}\right)=q_{n-k} \prod_{j=0}^{k-1} p_{n-j}, P\left(\bigcap_{i=1}^{n-k-1} E_{i}^{\prime}\right)=Y_{n-k-1}
$$

Equations (7), (10), and (11) therefore yield

$$
Y_{n}=\sum_{k=0}^{a-1} q_{n-k}\left(\prod_{j=0}^{k-1} p_{n-j}\right) Y_{n-k-1}+P\left(R_{n} \cap Q_{n-a}^{\prime} \cap \bigcap_{i=1}^{n-1} E_{i}^{\prime}\right) .
$$

Equation (12) indicates that further progress requires a decomposition of the event $Q_{n \cdot a}^{\prime} \cap \bigcap_{i=1}^{n-1} E_{i}^{\prime}$. As a first step in this direction, it will be shown that

$$
Q_{n-a}^{\prime} \subset E_{i}^{\prime} \quad \text { for } n-a \leq i \leq n-1,
$$

which implies that

$$
Q_{n-a}^{\prime} \cap \bigcap_{i=1}^{n-1} E_{i}^{\prime}=Q_{n-a}^{\prime} \cap \bigcap_{i=n-a}^{n-1} E_{i}^{\prime} \cap \bigcap_{i=1}^{n-a-1} E_{i}^{\prime}=Q_{n-a}^{\prime} \cap \bigcap_{i=1}^{n-a-1} E_{i}^{\prime}
$$

and thus that

$$
R_{n} \cap Q_{n-a}^{\prime} \cap \bigcap_{i=1}^{n-1} E_{i}^{\prime}=R_{n} \cap Q_{n-a}^{\prime} \cap \bigcap_{i=1}^{n-a-1} E_{i}^{\prime} .
$$

Since (a) $R_{n}$ is independent of $Q_{n-a}^{\prime}$ and also of $E_{i}^{\prime}$ for $i \leq n-a$, and (b) $P\left(R_{n}\right)=\underset{j=0}{a-1} \prod_{n-j}$, application of eq (14) to eq (12) yields

$$
Y_{n}=\sum_{k=0}^{a-1} q_{n-k}\left(\prod_{j=0}^{k-1} p_{n-j}\right) Y_{n-k-1}+\left(\prod_{j=0}^{a-1} p_{n-j}\right) P\left(Q_{n-a}^{\prime} \cap \bigcap_{i=1}^{n-a-1} E_{i}^{\prime}\right) \text {. }
$$

To prove (13), assume that $Q_{n-a}^{\prime}$ and $E_{i}$ both hold with $n-a \leq i \leq n-1$. Then $R_{i}$ holds, so that $i \geq a+b$ and the trials $T_{i}, T_{i-1}, \ldots, T_{i-a+1}$ all yield successes; in particular, this holds for the trials

$$
T_{n-a}, T_{n-1-a}, \ldots, T_{i+1-a} .
$$

If $i \leq n-b$, then $T_{n-a}, T_{n-a-1}, T_{n-a-2}, \ldots, T_{n-a-b+1}$ would be in the sequence (16) and therefore would all yield successes, contradicting the occurrence of $Q_{n-a}^{\prime}$. So $\imath>n-b$, i.e., $i-a>n-a-b>n-a-c$, a fact to be used below in (17), in the form $i-a \geq m(n-a)$.

Since $E_{i}$ occurs, $Q_{i-a}$ must occur, so that there are at least $b$ successes in the trials $T_{i-a}, T_{i-a-1}, \ldots, T_{m(i-a)}$. This last-mentioned sequence of trials can be split into two subsequences,

and

$$
T_{i-a}, T_{i-a-1}, \ldots, T_{m(n-a)},
$$

$$
T_{n-a-c}, T_{n-a-c-1}, \ldots, T_{m(i-a)}
$$

where the second subsequence is absent if $n \leq a+c$. Of the "at least $b$ successes" just mentioned, at most $n-i$ can occur in the subsequence (18), since the number of trials in this subsequence is at most

$$
n-a-c-(i-a-c+1)+1=n-i .
$$


Thus there are at least $b-(n-i)$ successes in the subsequence (17). Combining these with the $n-i$ successes in the sequence (16) (which is disjoint from (17)) yields at least $b$ successes in the sequence $T_{n-a}, T_{n-a-1}, \ldots, T_{m(n-a)}$, but this contradicts the occurrence of $Q_{n-a}^{\prime}$. Thus the assumption that $Q_{n-a}^{\prime}$ and $E_{i}$ both hold is untenable, and the inclusion (13) is proved.

Equation (15) shows that further progress requires a decomposition of the event $Q_{n-a}^{\prime} \cap \bigcap_{i=1}^{n-a-1} E_{i}^{\prime}$. This will first be done for $n \leq 2 a+c$, by showing that

$$
\begin{gathered}
Q_{a-n}^{\prime} \subseteq E_{i}^{\prime} \quad \text { for } 1 \leq i \leq n-a-1, \quad \text { if } a+b<n \leq a+c, \\
Q_{n-a}^{\prime} \subseteq E_{i}^{\prime} \quad \text { for } n-a-c+1 \leq i \leq n-a-1, \quad \text { if } a+c<n \leq 2 a+c .
\end{gathered}
$$

These results imply the decompositions

$$
\begin{gathered}
Q_{n-a}^{\prime} \cap \bigcap_{i=1}^{n-a-1} E_{i}^{\prime}=Q_{n-a}^{\prime} \quad \text { if } a+b<n \leq a+c, \\
Q_{n-a}^{\prime} \cap \bigcap_{i=1}^{n-a-1} E_{i}^{\prime}=Q_{n-a}^{\prime} \cap \bigcap_{i=1}^{n-a-c} E_{i}^{\prime} \quad \text { if } a+c<n \leq 2 a+c .
\end{gathered}
$$

Since $E_{i}$ cannot occur for $i<a$, and can occur for $i=a$ only when $b=0$, in which case we adopt the convention $c=0$, the last two displays can be rewritten as

$$
Q_{n-a}^{\prime} \cap \bigcap_{i=1}^{n-a-1} E_{i}^{\prime}=Q_{n-a}^{\prime} \quad \text { if } a+b<n \leq 2 a+c .
$$

Substitution of these results into eq (15) yields

$$
Y_{n}=\sum_{k=0}^{a-1} q_{n-k}\left(\prod_{j=0}^{k-1} p_{n-j}\right) Y_{n-k-1}+\left(\prod_{j=0}^{a-1} p_{n-j}\right) P\left(Q_{n-a}^{\prime}\right) \quad \text { for } a+b<n \leq 2 a+c .
$$

The evaluation of $P\left(Q_{n-a}^{\prime}\right)$, which appears in eq (24), will be discussed later.

To prove the inclusions (19) and (20), first note that both can be simultaneously written as

$$
Q_{n-a}^{\prime} \subseteq E_{i}^{\prime} \quad \text { for } m(n-a) \leq i \leq n-a-1, \quad \text { if } a+b<n \leq 2 a+c .
$$

Suppose, on the contrary, that both $Q_{n-a}^{\prime}$ and $E_{i}$ occur. Since $E_{i}$ occurs, there must be at least $a+b$ successes in the sequence of trials $T_{i}, T_{i-1}, \ldots, T_{m(i-a)}$. Since

$$
i \leq n-a-1 \leq(2 a+c)-a-1=a+c-1
$$

we have $m(i-a)=1$, and so there are at least $a+b$ successes among the trials $T_{i}, T_{i-1}, \ldots, T_{1}$. Since $Q_{n-a}^{\prime}$ holds, at most $b-1$ of these successes occur among trials $T_{i}, T_{i-1}, \ldots$. , $T_{m(n-a)}$, and so there are at least $a+b-(b-1)=a+1$ successes in trials $T_{m(n-a)-1}, T_{m(n-a)-2}, \ldots, T_{1}$. Thus

$$
a+1 \leq m(n-a)-1=\max (n-a-c, 0),
$$

implying $a+1 \leq n-a-c$ and thus $n>2 a+c$, a contradiction. So (19) and (20) are proved. Thus the desired recursion equation for the sequence $\left\{Y_{n}\right\}$ has been derived for $n \leq 2 a+c$, and is given by eqs (5), (6), and (24). From now on, $n>2 a+c$ is assumed.

To decompose $Q_{n-a}^{\prime} \cap \bigcap_{i=1}^{n-a-1} E_{i}^{\prime}$ when $n>2 a+c$, it is helpful to observe that $Q_{n-a}^{\prime}$ can be defined as the occurrence of at least $c-(b-1)=(c-b)+1$ failures in the $c$ trials between $T_{n-a}$ and $T_{n-a-c+1}$ inclusive. Not all of these failures can occur in the $c-b$ trials between $T_{n-a}$ and $T_{n-a-(c-b)+1}$ inclusive. We therefore introduce the new events defined for $0 \leq t \leq b-1$ by $F_{n t}$ : the $(c-b)+1$ st failure among $T_{n-a}, T_{n-a-1}, \ldots$,

$$
T_{n-a-c+1} \text { (in that order) occurs at } T_{n-a-(c-b)-\imath} \text {. }
$$

Then $Q_{n-a}^{\prime}=\sum_{t=0}^{b-1} F_{n t}$, which implies the decomposition 
so that eq (15) becomes

$$
Q_{n-a}^{\prime} \cap \bigcap_{i=1}^{n-a-1} E_{i}^{\prime}=\sum_{t=0}^{b-1}\left(F_{n \iota} \cap \bigcap_{i=1}^{n-a-1} E_{i}^{\prime}\right)
$$

$$
Y_{n}=\sum_{k=0}^{a-1} q_{n-k}\left(\prod_{j=0}^{k-1} p_{n-j}\right) Y_{n-k-1}+\left(\prod_{j=0}^{a-1} p_{n-j}\right) \sum_{t=0}^{b-1} P\left(F_{n t} \cap \bigcap_{i=1}^{n-a-1} E_{i}^{\prime}\right) .
$$

The solution under the extra hypothesis $a \geq b$ will now be completed by showing that

This will imply

$$
F_{n t} \subseteq E_{i}^{\prime} \quad \text { for } n-a-(c-b)-t \leq i \leq n-a-1, \quad \text { if } a \geq b .
$$

$$
F_{n t} \cap \bigcap_{i=1}^{n-a-1} E_{i}^{\prime}=F_{n t} \cap \bigcap_{i=1}^{n-a-(c-b)-t-1} E_{i}^{\prime}
$$

which, since $F_{n \imath}$ and $E_{i}^{\prime}$ are independent for $1 \leq i \leq n-a-(c-b)-t-1$, will turn eq (25) into

$$
Y_{n}=\sum_{k=0}^{a-1} q_{n-k}\left(\prod_{j=0}^{k-1} p_{n-j}\right) Y_{n-k-1}+\left(\prod_{j=0}^{a-1} p_{n-j}\right) \sum_{t=0}^{b-1} P\left(F_{n t}\right) Y_{n-a-(c-b)-t-1} \quad \text { if } a \geq b .
$$

Equation (27) is the desired recursion relation for $n>2 a+c$; together with eq (24) and the initial conditions of eqs (5) and (6), it entirely specifies the sequence $\left\{Y_{n}\right\}$ when $a \geq b$. The calculation of the coefficients $P\left(F_{n t}\right)$ will be discussed later.

To prove the inclusion (26), suppose that $F_{n t}$ and $E_{i}$ both hold, with $a \geq b$ and $n-a-(c-b)-t \leq i \leq n-a-1$. Since $i \geq n-a-(c-b)-t$, the (success-yielding) trials between $T_{i}$ and $T_{i-a+1}$ inclusive must have greater subscripts than the (failure-yielding) $T_{n-a-(c-b)-t}$, so that

$$
n-a-c+1 \leq n-a-(c-b)-t<i-a+1 \leq i \leq n-a-1
$$

so that the $a$ successes just mentioned fall between $T_{n-a}$ and $T_{n-a-c+1}$ inclusive. Because $a \geq b$, this implies that $Q_{n-a}$ occurs, contradicting the assumption that $F_{n t}$ occurs (recall that $\left.F_{n t} \subset Q_{n-a}^{\prime}\right)$.

The reasoning just employed carries over in part to the case $a \leq b$. Specifically, it will be shown that

$$
F_{n t} \supset E_{i}^{\prime} \quad \text { for } n-a-(c-b)-t \leq i \leq n-a-1 \text { and } t \leq a-1
$$

so that eq (25) becomes

$$
\begin{aligned}
Y_{n}=\sum_{k=0}^{a-1} q_{n-k}\left(\prod_{j=0}^{k-1} p_{n-j}\right) Y_{n-k-1}+\left(\prod_{j=0}^{a-1} p_{n-j}\right) \sum_{t=0}^{a-1} P\left(F_{n t}\right) Y_{n-a-(c-b)-t-1} & \\
& +\left(\prod_{j=0}^{a-1} p_{n-j}\right) \sum_{t=a}^{b-1} P\left(F_{n t} \cap \bigcap_{i=1}^{n-a-1} E_{i}^{\prime}\right) .
\end{aligned}
$$

To prove the inclusion (28), suppose that $F_{n t}$ and $E_{i}$ both hold. As in the last paragraph, $E_{i}$ requires at least $a$ successes between trials $T_{n-a}$ and $T_{n-a-(c-b)-t}$ inclusive, whereas $F_{n t}$ requires exactly $(c-b)+1$ failures (and thus exactly t successes) in these $(c-b)+t+1$ trials. Therefore, $a \leq t$, contrary to hypothesis in (28).

It is also easy to show that, for $a \leq t \leq b-1$,

$$
F_{n t} \subseteq E_{i}^{\prime} \quad \text { for } n-a-(c-b)-t \leq i \leq n-(c-b)-t-1,
$$

so that eq (29) becomes

$$
\begin{aligned}
Y_{n}=\sum_{k=0}^{a-1} q_{n-k}\left(\prod_{j=0}^{k-1} p_{n-j}\right) Y_{n-k-1} & +\left(\prod_{j=0}^{a-1} p_{n-j}\right) \sum_{t=0}^{a-1} P\left(F_{n t}\right) Y_{n-a-(c-b)-t-1} \\
& +\left(\prod_{j=0}^{a-1} p_{n-j}\right) \sum_{t=a}^{b-1} P\left(F_{n t} \cap \bigcap_{i=n-(c-b)-t}^{n-a-1} E_{i}^{\prime} \cap \bigcap_{i=1}^{n-a-(c-b)-t-1} E_{i}^{\prime}\right) .
\end{aligned}
$$


for if $F_{n t}$ and $E_{i}$ both occur, with $i \geq n-a-(c-b)-t$, then the fact that $T_{\imath}, T_{i-1}, \ldots ., T_{i-a+1}$ all yield successes whereas $T_{n-a-(c-b)-t}$ yields a failure, implies that

$$
i-a+1>n-a-(c-b)-t,
$$

i.e., $i \geq n-(c-b)-t$. The authors have not succeeded in carrying this analysis further in the case $a<b$.

\section{A Possible Simplification}

The recursion eq (27) is of order $a+c$, i.e., each member of the sequence $\left\{Y_{n}\right\}$ is expressed in terms of the $a+c$ members immediately preceding it. Actually, only $a+b$ of these $a+c$ preceding members are employed, ${ }^{9}$ the others appearing with zero coefficients in eq (27). In this section eq $(27)$ is used to derive a recursion equation of the higher degree $a+c+1$, which however has the advantage that calculation of a particular member of $\left\{Y_{n}\right\}$ requires only $b+3$ of the preceding $a+c+1$ members (and only 2 if $b=0$ ). Of course a detailed evaluation of this "advantage" would involve a comparison of the computational labor for the coefficients of eq (27) versus that for the coefficients of the new equation.

For the derivation, first change $n$ to $n+1$ in eq (27) and shift "dummy indices" to write the result, for $b>1$, as

$$
\begin{aligned}
Y_{n+1}= & q_{n+1} Y_{n}+\sum_{k=0}^{a-2} q_{n-k}\left(\prod_{j=0}^{k} p_{n+1-j}\right) Y_{n-k-1}+\left(\prod_{j=0}^{a-1} p_{n+1-j}\right)\left(\prod_{i=0}^{c-b} q_{n+1-a-i}\right) Y_{n-a-(c-b)} \\
& +\left(\prod_{j=0}^{a-1} p_{n+1-j}\right) P\left(F_{n+1,1}\right) Y_{n-a-(c-b)-1}+\left(\prod_{j=0}^{a-1} p_{n+1-j}\right) \sum_{i=1}^{b-2} P\left(F_{n+1, t+1}\right) Y_{n-a-(c-b)-t-1} .
\end{aligned}
$$

Next multiply eq (27) through by $p_{n+1}$, and write the result as

$$
\begin{aligned}
0=p_{n+1} Y_{n} & -\sum_{k=0}^{a-2} q_{n-k}\left(\prod_{j=0}^{k} p_{n+1-j}\right) Y_{n-k-1}-q_{n-a+1}\left(\prod_{j=0}^{a-1} p_{n+1-j}\right) Y_{n-a} \\
& -\left(\prod_{j=0}^{a} p_{n+1-j}\right)\left(\prod_{i=0}^{c-b} q_{n-a-i}\right) Y_{n-a-(c-b)-1}-\left(\prod_{j=0}^{a} p_{n+1-j}\right) \sum_{t=1}^{b-1} P\left(F_{n t}\right) Y_{n-a-(c-b)-t-1} .
\end{aligned}
$$

Now add eqs (32) and (33), noting that $p_{n+1}+q_{n+1}=1$ :

$$
\begin{aligned}
Y_{n+1}=Y_{n}-q_{n-a+1} & \left(\prod_{j=0}^{a-1} p_{n+1-j}\right) Y_{n-a}+\left(\prod_{j=0}^{a-1} p_{n+1-j}\right)\left(\begin{array}{c}
c-b \\
\prod_{i=0} q_{n+1-a-i}
\end{array}\right) Y_{n-a-(c-b)} \\
+ & \left(\prod_{j=0}^{a-1} p_{n+1-j}\right)\left[P\left(F_{n+1,1}\right)-p_{n+1-a}\left(\stackrel{c-b}{\left.\prod_{i=0}^{c} q_{n-a-i}\right)}\right)\right] Y_{n-a-(c-b)-1}, \\
+ & \left(\prod_{j=0}^{a-1} p_{n+1-j}\right) \sum_{t=1}^{b-2}\left[P\left(F_{n+1, t+1}\right)-p_{n+1-a} P\left(F_{n t}\right)\right] Y_{n-a-(c-b)-t-1} \\
& -\left(\prod_{j=0}^{a} p_{n+1-j}\right) P\left(F_{n, b-1}\right) Y_{n-a-c} .
\end{aligned}
$$

This is the desired equation.

A similar equation can be derived to replace eq (24). The procedure is as above, and the result is:

$$
Y_{n+1}=Y_{n}-q_{n-a+1}\left(\prod_{j=0}^{a-1} p_{n+1-j}\right) Y_{n-a}+\left(\prod_{j=0}^{a-1} p_{n+1-j}\right)\left[P\left(Q_{n+1-a}^{\prime}\right)-p_{n+1-a} P\left(Q_{n-a}^{\prime}\right)\right]
$$

for $a+b<n<2 a+c$. The analogous equation for the "transition case" $n=2 a+c$ will be omitted.

9 The order of the recursion is related to the "memory" requirements of a possible computer program, while the number of nonzero coefficients is relevant to the amount of computation required for each $Y_{n}$. 
If $b=0$ or $b=1$, the preceding equations must be modified. For $b=0$, eq (34) becomes

$$
Y_{n+1}=Y_{n}-q_{n-a+1}\left(\prod_{j=0}^{a-1} p_{n+1-j}\right) Y_{n-a},
$$

while for $b=1$ it becomes

$$
\begin{aligned}
Y_{n+1}=Y_{n}-q_{n-a+1}\left(\prod_{j=0}^{a-1} p_{n+1-j}\right) Y_{n-a}+\left(\prod_{j=0}^{a-1} p_{n+1-j}\right) & \left(\prod_{i=0}^{c-b} q_{n+1-a-i}\right) Y_{n-a-c+1} \\
& -\left(\prod_{j=0}^{a} p_{n+1-j}\right)\left(\prod_{i=0}^{c-b} q_{n-a-i}\right) Y_{n-a-c} .
\end{aligned}
$$

The analogous results for eq (34a) are omitted.

\section{A Special Case}

It is natural to inquire what form the recursion equations take in the relatively simple case when all the trials are governed by the same probability distribution, i.e.,

The initial conditions are

$$
p_{n}=p, q_{n}=q=1-p .
$$

$$
\begin{gathered}
Y_{n}=1 \quad \text { for } n<a+b, \\
Y_{a+b}=1-p^{a+b} .
\end{gathered}
$$

From the definition of $Q_{n-a}^{\prime}$ it follows that in eq (24)

$$
\begin{array}{ll}
P\left(Q_{n-a}^{\prime}\right)=\sum_{t=0}^{b-1}\left(\begin{array}{c}
n-a \\
t
\end{array}\right) p^{t} q^{n-a-t} & \text { for } a+b<n \leq a+c, \\
P\left(Q_{n-a}^{\prime}\right)=\sum_{t=0}^{b-1}\left(\begin{array}{l}
c \\
t
\end{array}\right) p^{t} q^{c-t} & \text { for } a+c<n \leq 2 a+c .
\end{array}
$$

Thus the early recursion equations read (for $q>0$ )

$$
\begin{array}{cc}
Y_{n}=q \sum_{k=0}^{a-1} p^{k} Y_{n-k-1}+p^{a} q^{n-a} \sum_{t=0}^{b-1}\left(\begin{array}{c}
n-a \\
t
\end{array}\right) p^{t} q^{-t} & \text { if } a+b<n \leq a+c, \\
Y_{n}=q \sum_{k=0}^{a-1} p^{k} Y_{n-k-1}+p^{a} q^{c}\left(\sum_{t=0}^{b-1}\left(\begin{array}{c}
c \\
t
\end{array}\right) p^{t} q^{-t}\right) Y_{n-a-c} & \text { if } a+c<n \leq 2 a+c .
\end{array}
$$

From the definition of $F_{n t}$, it follows that

$$
P\left(F_{n t}\right)=\left(\begin{array}{c}
c-b+t \\
t
\end{array}\right) p^{t} q^{c-b+1}
$$

so that eq (27), which is valid if $a \geq b$, becomes

$$
Y_{n}=q \sum_{k=0}^{a-1} p^{k} Y_{n-k-1}+p^{a} q^{c-b+1} \sum_{t=0}^{b-1}\left(\begin{array}{c}
c-b+t \\
t
\end{array}\right) p^{t} Y_{n-a-(c-b)-t-1} \quad \text { if } n>2 a+c,
$$

while eq (34) becomes (for $b>1$ )

$$
\begin{aligned}
& Y_{n+1}=Y_{n}-q p^{a} Y_{n-a}+p^{a} q^{c-b+1} Y_{n-a-(c-b)}+(c-b) p^{a+1} q^{c-b+1} Y_{n-a-(c-b)-1} \\
& \quad+p^{a+1} q^{c-b+1} \sum_{t=1}^{b-2}\left(\begin{array}{c}
c-b+t \\
t+1
\end{array}\right) p^{t} Y_{n-a-(c-b)-t-1}-p^{a+b} q^{c-b+1}\left(\begin{array}{c}
c-1 \\
b-1
\end{array}\right) Y_{n-a-c} .
\end{aligned}
$$

Let the random variable $N$ be defined by the condition that $T_{N}$ is the last trial of the first run immediately preceded by a quota. The sequence $\left\{X_{n}\right\}$ defined in eq (2) is thus 
precisely the frequency distribution function of $N$. Although the equations above are not spectacularly simple despite the simple choice of the sequence $\left\{p_{n}\right\}$, they at least permit description of a finite procedure for calculating the expected value $E(N)$ of $N$ in the special case under consideration (with $a \geq b$ ).

To this end, first use eq (4) to write

$$
\begin{aligned}
E(N)=\sum_{1} n X_{n} & =X_{1}+\sum_{2} n\left(Y_{n-1}-Y_{n}\right) \\
& =\left(1-Y_{1}\right)+\lim _{m \rightarrow \infty}\left(\sum_{2}^{m} n Y_{n-1}-\sum_{2}^{m} n Y_{n}\right) \\
& =1+\lim _{m \rightarrow \infty}\left(\sum_{1}^{m-1} Y_{n}-m Y_{m}\right) .
\end{aligned}
$$

Since the definition (3) of the sequence $\left\{Y_{n}\right\}$ ensures that this is a monotone nonascending sequence of nonnegative terms, a classical theorem on infinite series ${ }^{10}$ can be invoked to assert that $m Y_{m} \rightarrow 0$ if $\sum_{1} Y_{n}<\infty$. This yields

$$
E(N)=1+\sum_{1} Y_{n} \quad \text { if } \sum_{1} Y_{n}<\infty,
$$

so that the evaluation of $E(N)$ reduces to that of $\sum_{1} Y_{n}$. This of course holds even in the general case.

We now employ the standard formal procedure for summing an infinite series whose terms obey a linear difference equation with constant coefficients. Write eq (39) briefly as

$$
Y_{n}=\sum_{i=1}^{a+c} a_{i} Y_{n-i} \quad \text { for } n>2 a+c,
$$

and introduce the generating function

as well as the polynomial

$$
g(z)=\sum_{1} Y_{n} z^{n}
$$

$$
f(z)=1-\sum_{i=1}^{a+c} a_{i} z^{i}
$$

An elementary calculation using eq (42) yields

$$
\begin{aligned}
f(z) g(z) & =\sum_{1} Y_{n} z^{n}-\sum_{i=1}^{a+c} a_{i} \sum_{1} Y_{n} z^{n+i} \\
& =\sum_{1} Y_{n} z^{n}-\sum_{i=1}^{a+c} a_{i} \sum_{n=i+1} Y_{n-i} z^{n} \\
& =\sum_{1} Y_{n} z^{n}-\sum_{n=2}\left(z^{n} \sum\left\{a_{i} Y_{n-i}: 1 \leq i \leq \min (a+c, n-1)\right\}\right) \\
& =1+\sum_{2}^{a+c}\left(Y_{n}-\sum_{i=1}^{n-1} a_{i} Y_{n-i}\right) z^{n}+\sum_{a+c+1}\left(Y_{n}-\sum_{i=1}^{a+c} a_{i} Y_{n-i}\right) z^{n} \\
& =1+\sum_{2}^{a+c}\left(Y_{n}-\sum_{i=1}^{n-1} a_{i} Y_{n-i}\right) z^{n}+\sum_{a+c+1}^{2 a+c}\left(Y_{n}-\sum_{i=1}^{a+c} a_{i} Y_{n-i}\right) z^{n}=h(z),
\end{aligned}
$$

where the coefficients of $h(z)$ can be found from eq (35) to (38).

It will be shown that for $0<p<1$

$$
f(z) \neq 0 \quad \text { for }|z| \leq 1 .
$$

10 See p. 124 of Knopp, Theory and Application of Infinite Series, Blackie (1951 edition). 
This justifies the preceding formal manipulations for $|z| \leq 1$, permits solution of eq (45) as $g(z)=h(z) / f(z)$, and permits use of eq (43) to write $\sum_{1} Y_{n}=g(1)$, so that eq (41) becomes

$$
E(N)=1+h(1) / f(1),
$$

the promised formula for $E(N)$. In connection with eq (47), note that by eqs (39) and (44)

$$
f(1)=p^{a}\left(1-q^{c-b+1} \sum_{t=0}^{b-1}\left(\begin{array}{c}
c-b+t \\
t
\end{array}\right) p^{t}\right) .
$$

To prove the nonequality (46), note that all $a_{i}$ in eqs (42) and (39) are nonnegative, so that for $|z| \leq 1$,

$$
\left|\sum_{i=1}^{a+c} a_{i} z^{i}\right| \leq \sum_{i=1}^{a+c} a_{i}|z|^{i} \leq \sum_{i=1}^{a+c} a_{i}
$$

thus (46) is true if $\sum_{i=1}^{a+c} a_{i}<1$, i.e., if $f(1)>0$. By eq (48) this is equivalent to

$$
\sum_{t=0}^{b-1}\left(\begin{array}{c}
c-b+t \\
t
\end{array}\right) p^{t} q^{c-b+1}<1,
$$

which is true since the left-hand sum is the value at $b-1$ of the cumulative negative binomial probability distribution with parameters $c-b+1$ and $q$. (See Feller, op. cit. in footnote 5.)

As in section 4 , the cases $b=0$ and $b=1$ require special treatment, which is left to the reader.

\section{Auxiliary Probabilities}

This section deals with the probabilities $P\left(Q_{n-a}^{\prime}\right)$, which appear in eq $(24)$, and $P\left(F_{n t}\right)$, which appear in eq (27). The "best" way of calculating these quantities (or more generally, of arranging the whole recursive computation) is likely to depend strongly on the values of $a, b, c$, on the peculiarities of the specific sequence $\left\{p_{n}\right\}$ of success probabilities under consideration, and perhaps on special characteristics of the calculating equipment used. Thus the procedure sketched below is illustrative, rather than "recommended."

For $a+b<n \leq a+c, Q_{n-a}^{\prime}$ is the event that the sequence of $n-a$ trials $T_{n-a}, T_{n-a-1}, \ldots$, $T_{1}$ yield at least $n-a-(b-1)$ failures. Not all of these failures can occur in the $n-a-b$ trials between $T_{n-a}$ and $T_{b+1}$, respectively. We therefore introduce new events defined for $0 \leq t \leq b-1$ by

$G_{n t}$ : the $n-a-(b-1)^{\text {st }}$ failure among $T_{n-a}, T_{n-a-1}, \ldots, T_{1}$ (in that order) occurs at $T_{b-t}$. Then

$$
\begin{aligned}
Q_{n-a}^{\prime} & =\sum_{t=0}^{b-1} G_{n t}, \text { so that } \\
P\left(Q_{n-a}^{\prime}\right) & =\sum_{t=0}^{b-1} P\left(G_{n t}\right) \quad \text { for } a+b<n \leq a+c .
\end{aligned}
$$

As in the analysis before eq (25),

$$
P\left(Q_{n-a}^{\prime}\right)=\sum_{t=0}^{b-1} P\left(F_{n t}\right) \quad \text { for } a+c<n \leq 2 a+c .
$$

The events $F_{n t}$ and $G_{n t}$ are special cases of the event $H_{n k t}$ : the $k$ th failure among $T_{n}, T_{n-1}, \ldots, T_{1}$ (in that order) occurs at $T_{n-k+1-t}$, defined for $0 \leq t \leq b-1$ and $1 \leq k \leq n-b+2$. Specifically, 


$$
G_{n t}=H_{n-a, n-a-b+1, t}, F_{n t}=H_{n-a, c-b+1, t}
$$

Thus it suffices to consider the probabilities $P\left(H_{n k t}\right)$. Now introduce the event

$$
J_{n k t} \text { : precisely } k-1 \text { failures between } T_{n} \text { and } T_{n-k+2-t} \text { inclusive. }
$$

Then clearly $P\left(H_{n 10}\right)=q_{n}$ and $P\left(H_{n k t}\right)=q_{n-k+1-t} P\left(J_{n k t}\right)$ otherwise, so it suffices to consider the probabilities $P\left(J_{n k t}\right)$ for $(k, t) \neq(1,0)$.

These probabilities can be computed by recursion on $k$ and $t$ after disposing of the case $k=1$ by

$$
P\left(J_{n 1 t}\right)=\prod_{i=0}^{t-1} p_{n-i} \quad \text { for } t>0
$$

The initiation of the recursion, for fixed $k>1$, is

$$
P\left(J_{n k 0}\right)=\stackrel{k-2}{=} \prod_{i=0} q_{n-i} \quad \text { for } k>1,
$$

and the "recursive step" is

$$
P\left(J_{n k t}\right)=p_{n-k+2-t} P\left(J_{n, k, t-1}\right)+q_{n-k+2-t} P\left(J_{n, k-1, t-1}\right) \quad \text { for } k>1, t>1 .
$$

\section{A Related Problem}

Let $K_{n}$ be the event that $T_{n}$ is the last of a sequence of $a+b$ consecutive trials all yielding successes. That is,

$$
K_{n}=\bigcap_{i=0}^{a+b-1} S_{n-i}
$$

For the application which motivated this paper, it is of interest to consider the conditional probabilities

$$
Z_{n}=P\left(K_{n} / E_{n} \cap \bigcap_{i=1}^{n-1} E_{i}^{\prime}\right)
$$

defined for $n \geq a+b$. From the definition of conditional probability, we have ${ }^{11}$

$$
X_{n} Z_{n}=P\left(K_{n} \cap E_{n} \cap \bigcap_{i=1}^{n-1} E_{i}^{\prime}\right),
$$

which because of the inclusion $K_{n} \subseteq E_{n}$ can be rewritten

$$
X_{n} Z_{n}=P\left(K_{n} \cap \bigcap_{i=1}^{n-1} E_{i}^{\prime}\right)
$$

Since the preceding material in principle permits the evaluation of $X_{n}$ when $a \geq b$, we need only investigate the (unconditional) probability on the right-hand side of eq (53). It will be assumed that $n>a+c+1$ and $b>0$.

It will be shown below that

$$
K_{n} \cap E_{n-a}^{\prime}=K_{n} \cap \bigcap_{i=n-a-c}^{n-a-b} S_{i}^{\prime}
$$

which implies that

$$
K_{n} \cap \bigcap_{i=1}^{n-1} E_{i}^{\prime}=K_{n} \cap \bigcap_{i=n-a-c}^{n-a-b} S_{i}^{\prime} \cap \bigcap_{i=1}^{n-2} E_{i}^{\prime} .
$$

\footnotetext{
11 Recall the definition of $X_{n}$ in eq (2).
} 
Since $\mathrm{S}_{i}^{\prime} \subseteq E_{i}^{\prime}$, the last equation can be rewritten

$$
K_{n} \cap \bigcap_{i=1}^{n-1} E_{i}^{\prime}=K_{n} \cap \bigcap_{i=n-a-c}^{n-a-b} S_{i}^{\prime} \cap \bigcap_{i=n-a-b+1}^{n-2} E_{i}^{\prime} \cap \bigcap_{i=1}^{n-a-c-1} E_{i}^{\prime}
$$

To prove eq (54), first assume its left-hand event occurs. Tentatively suppose $S_{i}$ occurs, with $n-a-c \leq i \leq n-a-b$. Since $K_{n}$ occurs, trials $T_{n-1}$ through $T_{n-a}$ all yield successes (i.e., $R_{n-1}$ holds), and in addition the $b-1$ trials $T_{n-a-1}$ through $T_{n-a-b+1}$ yield $b-1$ successes. These $(b-1)$ successes, together with $S_{i}$, occur between $T_{n-a-1}$ and $T_{n-a-c}$ inclusive, and so $Q_{n-1-a}$ holds. Thus $E_{n-1}=R_{n-1} \cap Q_{n-1-a}$ holds, contradicting the assumption that the lefthand event in eq (54) holds. So the tentative supposition is untenable, and the left-hand event is contained in (i.e., implies) the right-hand one. Conversely, if the right-hand event holds then trials $T_{n-a-b}$ through $T_{n-a-c}$ inclusive constitute $(c-b)+1$ failures between $T_{n-a-1}$ and $T_{n-a-c}$ inclusive, so that $Q_{n-1-a}^{\prime}$, and thus $E_{n-1}^{\prime}$, must hold. This shows that the righthand event contains the left-hand one, and so eq (54) is proved.

Next it will be shown that

$$
\bigcap_{i=n-a-c}^{n-a-b} S_{i}^{\prime} \subset E_{i}^{\prime} \quad \text { for } n-a-b+1 \leq i \leq n-2 .
$$

This implies that eq (55) can be rewritten

$$
K_{n} \cap \bigcap_{i=1}^{n-1} E_{i}^{\prime}=K_{n} \cap \bigcap_{i=n-a-c}^{n-a-b} S_{i}^{\prime} \cap \bigcap_{i=1}^{n-a-c-1} E_{i}^{\prime}
$$

and since the three events on the right-hand side are independent, eq (53) becomes

$$
\begin{aligned}
X_{n} Z_{n} & =P\left(K_{n}\right) P\left(\bigcap_{i=n-a-c}^{n-a-b} S_{i}^{\prime}\right) P\left(\bigcap_{i=1}^{n-a-c-1} E_{i}^{\prime}\right) \\
& =\left(\prod_{i=0}^{a+b-1} p_{n-i}\right)\left(\prod_{i=a+b}^{a+c} q_{n-i}\right) Y_{n-a-c-1},
\end{aligned}
$$

thus completing the solution.

To prove the inclusion (56), suppose its left-hand event occurs, and assume $n-a-b+$ $1 \leq i \leq n-2$. Tentatively assume $E_{i}$ occurs. Then $T_{i}$ through $T_{i-a+1}$ inclusive must yield successes, and so (since $i>n-a-b$ ) must have subscripts greater than the failure-yielding $T_{n-a-b}$. Thus $i-a+1>n-a-b$, and so $n-b \leq i \leq n-2$. This implies that

$$
i-a-c+1<n-a-c<n-a-b \leq i-a,
$$

so that the $(c-b)+1$ failure-yielding trials $T_{n-a-b}$ through $T_{n-a-c}$ inclusive lie between $T_{i-a}$ and $T_{i-a-c+1}$ inclusive, thus ensuring that $Q_{i-a}^{\prime}$ holds. Thus $E_{i}^{\prime}$ holds, contradicting the tentative assumption, which is therefore untenable. Therefore (56) is proved.

Suppose for example that $b=1$ and $c=2$. For $n>a+c+1=a+3$, eq (57) yields

$$
X_{n} Z_{n}=\left(\prod_{i=0}^{a} p_{n-i}\right) q_{n-a-1} q_{n-a-2} Y_{n-a-3} .
$$

On the other hand, if $n>2 a+3$ then the last equation of section 4 , with $n$ replaced by $n-1$, yields

$$
\begin{aligned}
& X_{n}=Y_{n-1}-Y_{n}=q_{n-a}\left(\prod_{j=0}^{a-1} p_{n-j}\right) Y_{n-a-1}-\left(\prod_{j=0}^{a-1} p_{n-j}\right) q_{n-a} q_{n-a-1} Y_{n-a-2} \\
&+\left(\prod_{j=0}^{a} p_{n-j}\right) q_{n-a-1} q_{n-a-2} Y_{n-a-3} .
\end{aligned}
$$


Comparison of this result with eq (58) shows that

$$
q_{n-a}\left(\prod_{j=0}^{a-1} p_{n-j}\right) Y_{n-a-1}-\left(\prod_{j=0}^{a-1} p_{n-j}\right) q_{n-a} q_{n-a-1} Y_{n-a-2}=\left(1-Z_{n}\right) X_{n}
$$

and thus that

$$
\left(1-Z_{n}\right) / Z_{n}=q_{n-a}\left(Y_{n-a-1}-q_{n-a-1} Y_{n-a-2}\right) / p_{n-a} q_{n-a-1} q_{n-a-2} Y_{n-a-3} .
$$

If $n>3 a+3$, however, then eq (27), with $n$ replaced by $n-a-1$, yields (for $a>1$ )

$$
\begin{aligned}
Y_{n-a-1}-q_{n-a-1} Y_{n-a-2}=p_{n-a-1} q_{n-a-2}+\sum_{k=2}^{a-1} q_{n-a-1-k} & \left(\prod_{j=0}^{k-1} p_{n-a-1-j}\right) Y_{n-a-k-2} \\
& +\left(\prod_{j=0}^{a-1} p_{n-a-j-1}\right) q_{n-2 a-1} q_{n-2 a-2} Y_{n-2 a-3} .
\end{aligned}
$$

On the one hand, eq (60) shows that

so that eq (59) yields

$$
Y_{n-a}-q_{n-a-1} Y_{n-a-2} \geq p_{n-a-1} q_{n-a-2} Y_{n-a-3}
$$

$$
\left(1-Z_{n}\right) / Z_{n} \geq q_{n-a} p_{n-a-1} / p_{n-a} q_{n-a-1}
$$

This shows, e.g., that if the sequence $\left\{p_{n}\right\}$ is nonincreasing for all $n>n_{0}>2 a+3$, then $Z_{n} \leq 1 / 2$ for all $n>n_{0}+a$. A bit less crudely, since the sequence $\left\{Y_{n}\right\}$ is nonincreasing by its definition, eq $(60)$ yields

$$
\begin{aligned}
& Y_{n-a}-q_{n-a-1} Y_{n-a-2} \geq p_{n-a-1} Y_{n-a-3}\left\{q_{n-a-2}+\sum_{k=2}^{a-1} q_{n-a-1-k}\left(\prod_{j=1}^{k-1} p_{n-a-1-j}\right)\right. \\
&\left.+\left(\prod_{j=1}^{a-1} p_{n-a-j-1}\right) q_{n-2 a-1} q_{n-2 a-2}\right\},
\end{aligned}
$$

from which a more precise lower bound on $\left(1-Z_{n}\right) / Z_{n}$ can be derived via eq (59). On the other hand, since $Y_{m} \geq q_{m} Y_{m-1}$, we have

$$
\begin{aligned}
& Y_{n-a-k-2} \leq Y_{n-a-3} \int_{i=3}^{k+1} q_{n-a-i}, \\
& Y_{n-2 a-3} \leq Y_{n-a-3} \prod_{i=3}^{a+2} q_{n-a-i},
\end{aligned}
$$

which when substituted into eq (60) yield an inequality that can be combined with eq (59) to obtain a crude upper bound on $\left(1-Z_{n}\right) / Z_{n}$.

If $a=1$ (as well as $b=1$ and $c=2$ ) then eq (27) yields

so that eq (59) becomes

$$
Y_{n-2}-q_{n-2} Y_{n-3}=p_{n-2} q_{n-3} q_{n-4} Y_{n-5}
$$

$$
\left(1-Z_{n}\right) / Z_{n}=q_{n-1} p_{n-2} q_{n-4} Y_{n-5} / p_{n-1} q_{n-2} Y_{n-4} .
$$

Since $q_{n-4} Y_{n-5} \leq Y_{n-4} \leq Y_{n-5}$, we have

$$
q_{n-1} p_{n-2} q_{n-4} / p_{n-1} q_{n-2} \leq\left(1-Z_{n}\right) / Z_{n} \leq q_{n-1} p_{n-2} / p_{n-1} q_{n-2} .
$$

In particular, if $\left\{p_{n}\right\}$ is ultimately nondecreasing then ultimately $Z_{n} \geq 1 / 2$. 